\title{
Significance of DEK overexpression for the prognostic evaluation of non-small cell lung carcinoma
}

\author{
$\mathrm{XIN} \mathrm{LIU}^{1 *}$, DONGDONG $\mathrm{QI}^{2 *}, \mathrm{JUJIE}^{3}{ }^{3}, \mathrm{ZESHU}_{\mathrm{MAO}}^{2}, \mathrm{XIANGDAN} \mathrm{LI}{ }^{4}$, \\ JINHUI ZHANG ${ }^{1}$, JINZI LI ${ }^{4}$ and WENBIN GAO ${ }^{2,5}$ \\ ${ }^{1}$ Institute of Molecular Medicine, Medical College of Eastern Liaoning University, Dandong, Liaoning 118000; \\ ${ }^{2}$ Department of Oncology, Affiliated Zhongshan Hospital of Dalian University, Dalian, Liaoning 116000; \\ ${ }^{3}$ Traditional Chinese Medical Hospital of Anqiu City, Anqiu, Shandong 262100; \\ ${ }^{4}$ Department of Pediatrics, Yanbian University Hospital, Yanji, Jilin 133002; \\ ${ }^{5}$ Department of Oncology, Shenzhen Luohu People's Hospital, Shenzhen 518000, P.R. China
}

Received July 12, 2015; Accepted September 3, 2015

DOI: $10.3892 / o r .2015 .4365$

\begin{abstract}
In the present study, we explored the role of DEK expression for the prognostic evaluation of non-small cell lung carcinoma (NSCLC). DEK protein and mRNA expression levels were detected in NSCLC cells and fresh tissue samples of NSCLC paired with adjacent non-tumor tissues, respectively. NSCLC cases $(n=196)$ meeting strict follow-up criteria were selected for immunohistochemical staining of DEK protein. Correlations between DEK expression and clinicopathological features of the NSCLC cases were evaluated using Chi-square tests. Survival rates were calculated using the Kaplan-Meier method, and the relationship between prognostic factors and patient overall survival was analyzed using Cox proportional hazard analysis. Based on the results, the levels of DEK protein and mRNA were significantly upregulated in 6 fresh tissue samples of NSCLC. Immunohistochemical analysis showed that the DEK expression rate was significantly higher in the NSCLC samples compared with either the adjacent non-tumor tissues or normal lung tissues. DEK expression was correlated with poor differentiation and late pathological stage of NSCLC. DEK expression was also correlated with low disease-free survival and overall survival rates. In the earlystage group, disease-free and overall survival rates of patients with DEK expression were significantly lower than those of
\end{abstract}

Correspondence to: Dr Jinzi Li, Department of Pediatrics, Yanbian University Hospital, No. 1327 Juzi Street, Yanji, Jilin 133002, P.R. China

E-mail: 1559468491@qq.com

Dr Wenbin Gao, Department of Oncology, Shenzhen Luohu People's Hospital, Shenzhen 518000, P.R. China

E-mail: 1453371147@qq.com

*Contributed equally

Key words: DEK, non-small cell lung carcinoma, prognosis, survival analysis patients without DEK expression. Further analysis using a Cox proportional hazard regression model revealed that DEK expression emerged as a significant independent hazard factor for the overall survival rate of patients with NSCLC. Consequently, DEK plays an important role in the progression of NSCLC. DEK may potentially be used as an independent biomarker for the prognostic evaluation of NSCLC.

\section{Introduction}

Lung cancer is a leading cause of cancer-related deaths worldwide with over one million cases diagnosed yearly (1). Lung cancer is morphologically divided into non-small cell lung carcinoma (NSCLC) and small cell lung carcinoma, in which NSCLC accounts for $\sim 80 \%$ of all lung cancer cases $(2,3)$. Despite progress in the multimodality treatment of lung cancer, prognosis is still poor with a $10-15 \%$ 5-year survival rate. Therefore, the identification of a reliable biomarker for predicting recurrence and for identifying tumors is important not only for understanding the molecular and cellular processes involved, but also for searching for possible new therapeutic molecular targets.

The proto-oncogene protein DEK was originally identified as a fusion with the CAN/NUP214 nucleoporin in a subset of acute myeloid leukemia patients $(4,5)$. DEK is abundantly expressed in proliferating cells, and the majority of the protein is bound to chromatin, whereas a small fraction is bound to RNA. The 43-kDa nuclear phosphoprotein is the only member of its family, and contains a conserved central SAP DNA binding domain with homology to SAF-A/B, acinus and PIAS, and a second DNA binding motif within the C-terminus. Studies have suggested that DEK may promote tumorigenesis, at least in part, by its ability to interfere with cell division, DNA repair, inhibit cell differentiation, senescence and apoptosis, and cooperate with transforming oncogenes (6-9). Wise-Draper et al recently reported that DEK expression promoted transformation in vitro and in vivo and the DEK proto-oncogene is upregulated in many human cancers including colon, breast, ovarian and cervical cancer (5). The degree of DEK upregulation often correlates 
to the severity of prognosis as indicated by histopathological determination of a later stage and grade, or poor differentiation characteristics $(5,10)$. Our previous studies showed that DEK protein was closely related to the proliferation of serous ovarian tumor cells and an increased proliferating index of Ki-67 in cervical cancer $(11,12)$. Furthermore, based on tumor tissue analyses, we found that DEK expression was correlated with the prognosis of a variety of human tumors, such as breast and colorectal cancer $(13,14)$. Thus, DEK is expected to be the new molecular target for cancer therapy. However, the role of DEK in the prognostic evaluation and its relationship to survival in NSCLC are unknown. The critical role of DEK in numerous cancers impelled us to study the function of DEK in NSCLC. Therefore, we performed immunofluorescence (IF) staining in NSCLC A549 cells, and quantitative real-time RT-PCR (qRT-PCR), western blotting and immunohistochemical (IHC) staining of DEK in NSCLC and normal lung tissues, and found that DEK protein was usually upregulated in NSCLC compared with the normal counterparts. Multivariate analysis revealed that DEK may be an independent biomarker for predicting NSCLC prognosis.

\section{Materials and methods}

Ethics statement. The present study complied with the Helsinki Declaration and was approved by the Human Ethics and Research Ethics Committees of the Medical College of Eastern Liaoning University in China. Through the surgery consent form, patients were informed that the resected specimens would be stored by the hospital and potentially used for scientific research, and that their privacy would be maintained. Follow-up survival data were retrospectively collected through medical-record analyses.

IF staining for DEK protein in non-small cell lung cancer cells (A549). The lung cancer cell line, A549, was grown on coverslips to $70 \%$ confluency and then fixed in $4 \%$ paraformaldehyde for $10 \mathrm{~min}$ and permeabilized with $0.5 \%$ Triton $\mathrm{X}-100$ for 10 min after $24 \mathrm{~h}$. Blocking was performed with $3 \%$ bovine serum albumin fraction V (Solarbio, Beijing, China) for $1 \mathrm{~h}$ at room temperature. After washing with phosphate-buffered saline (PBS), the cells were incubated with mouse anti-human DEK (1:50; BD Biosciences Pharmingen, San Diego, CA, USA) at $4^{\circ} \mathrm{C}$ overnight, followed by incubation with Alexa Fluor 568 goat anti-mouse IgG (H+L) (A11004, 1:1,000; Life Technologies, Carlsbad, CA, USA) for $1 \mathrm{~h}$ at room temperature. After washing with PBS, the cells were counterstained with 4',6-diamidino-2-phenylindole (DAPI), and the coverslips were mounted with Antifade Mounting Medium (both from Beyotime, Shanghai, China). Finally, immunofluorescence signals were visualized and recorded using Leica SP5 II confocal microscope (21).

Clinical samples. Fresh samples from 6 cases of NSCLC were paired with adjacent non-cancerous tissues, and 196 cases of routinely processed and paraffin-embedded NSCLC meeting strict follow-up criteria were randomly selected from patients undergoing surgery between 2004 and 2008 at the Department of Pathology and Tumor Tissue Bank, the Medical College of Eastern Liaoning University. Pathological parameters, including age, gender, smoking status, tumor size, pathological stage, differentiation, subtype, CEA level, metastasis status, disease-free and overall survival data, were carefully reviewed. The patient ages ranged between 34 and 76 years, with a mean age of 64.6 years. The male to female ratio was 109:87. Tumors were staged according to the 6th edition of the American Joint Committee on Cancer (15). Of the 196 NSCLC samples, 101 were determined as early-stage (I-II) and 95 as late-stage (III-IV). Forty-four samples were well differentiated, 94 were moderately differentiated and 58 were poorly differentiated cancers. No patients had received chemotherapy and radiotherapy before surgery. By March 2013, 78 patients had died and 118 patients remained alive. The median survival time was 71 months.

Western blotting. Fresh tissue samples of NSCLC were ground to powder in liquid nitrogen and lysed with SDS-PAGE sample buffer. Equal protein samples $(20 \mu \mathrm{g})$ were separated on $12 \%$ SDS polyacrylamide gels and transferred to PVDF membranes. The membranes were blocked with $5 \%$ fat-free milk in tris-buffered saline containing $0.1 \%$ Tween-20 for $1 \mathrm{~h}$ at room temperature. The membranes were incubated with the DEK antibody (1:1,000; BD Biosciences Pharmingen) overnight at $4^{\circ} \mathrm{C}$, and then with horseradish peroxidase-conjugated rabbit anti-mouse IgG. DEK expression was detected using ECL Prime western blotting detection reagent (Amersham) according to the manufacturer's instructions. $\beta$-actin (Sigma, St. Louis, MO, USA) was used as a loading control. Protein bands were quantified using a LANE 1D system (Sage, China).

RNA extraction and $q R T-P C R$. Total RNA from fresh tissues was extracted using TRIzol reagent (Invitrogen, Carlsbad, CA, USA). First-strand cDNA was synthesized by PrimeScript reverse transcriptase (Takara Biotechnology, Dalian, China) and oligo(dT) following the manufacturer's instructions. To examine expression, real-time PCR was performed with a Bio-Rad sequence detection system according to the manufacturer's instructions using a double-stranded DNA-specific SYBR Premix Ex Taq ${ }^{\mathrm{TM}}$ II kit (Takara Biotechnology). Double-stranded DNA-specific expression was tested using the comparative $\mathrm{Ct}$ method using $2^{-\Delta \Delta \mathrm{Ct}}$. The DEK primers were: 5'-AAACCTAGCCAGCTTCACGA-3' and 5'-AGCCCC AACTCCAGAGAAAC-3'; GAPDH, 5'-GGTCTCCTCTGA CTTCAACA-3' and 5'-ATACCAGGAAATGAGCTTGA-3'. All assays were performed in triplicate and repeated at least three times.

Immunohistochemical analysis. Immunohistochemical analysis was performed using a Dako LSAB kit (Dako A/S, Glostrup, Denmark). Briefly, to eliminate endogenous peroxidase activity, $4-\mu \mathrm{m}$ thick tissue sections were deparaffinized, rehydrated and incubated with $3 \% \mathrm{H}_{2} \mathrm{O}_{2}$ in methanol for $15 \mathrm{~min}$ at room temperature. The antigen was retrieved at $95^{\circ} \mathrm{C}$ for $20 \mathrm{~min}$ by placing the slides in $0.01 \mathrm{M}$ sodium citrate buffer (pH 6.0). The slides were then incubated with the DEK antibody (1:50) at $4^{\circ} \mathrm{C}$ overnight. After incubation with the biotinylated secondary antibody at room temperature for $30 \mathrm{~min}$, the slides were incubated with streptavidin-peroxidase complex at room temperature for $30 \mathrm{~min}$. Immunostaining was developed using 3,3'-diaminobenzidine, and Mayer's hematoxylin was used for 

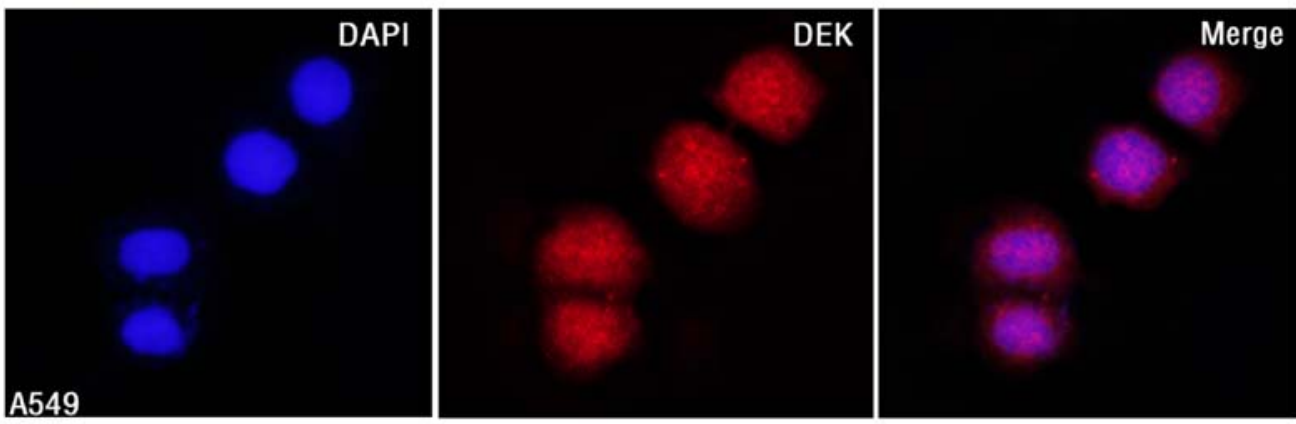

Figure 1. IF staining for DEK protein in A549 cells. DEK localized to the nuclei of A549 cells by IF staining.

A
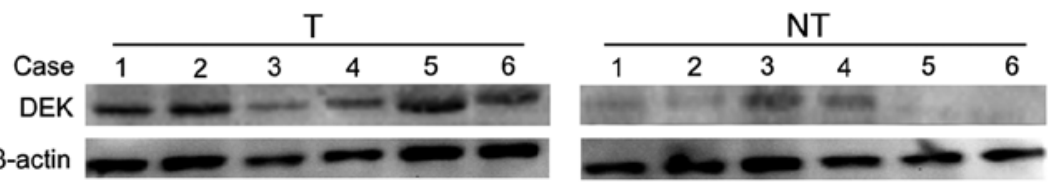

B

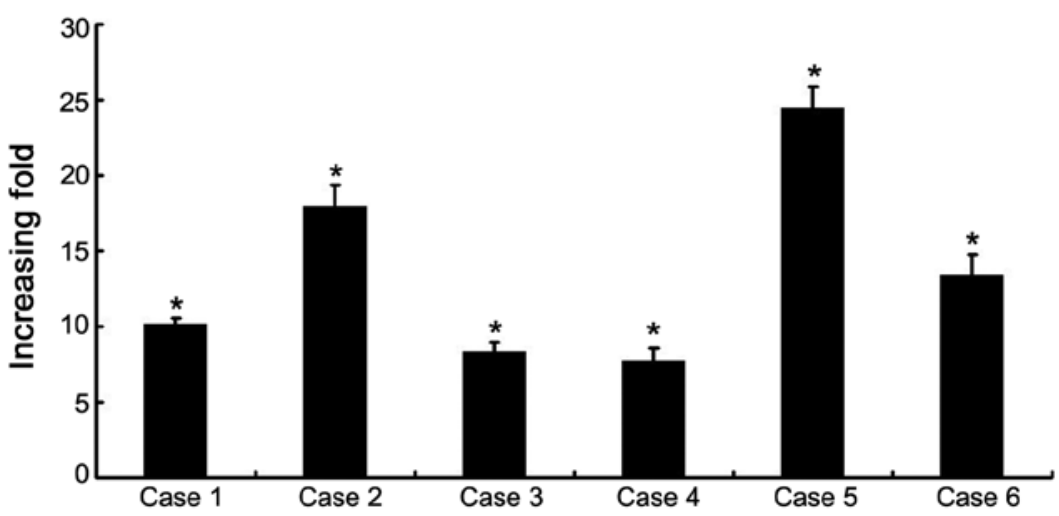

Figure 2. Overexpression of DEK protein in fresh NSCLC tissues. (A) Images of western blot analyses of DEK protein expression in 6 matched pairs of NSCLC (T) and adjacent non-tumor tissues (NT). (B) Relative T/NT ratios of DEK protein expression level in paired NSCLC (T) and adjacent non-tumor (NT) tissues (increasing fold-change, ${ }^{*} \mathrm{P}<0.05$ ).

counterstaining. Tonsil sections were used as positive controls and mouse $\mathrm{IgG}$ as isotope controls. For negative controls, positive tissue sections were processed in the same manner but the primary antibody (mouse anti-DEK) was omitted.

All specimens were examined by two pathologists (Z. Lin and S. Liu) who did not possess knowledge of the clinical data. In case of discrepancies, a final score was established by reassessment on a double-headed microscope. Briefly, immunostaining for DEK was semi-quantitatively scored as '-' (none or $<5 \%$ positive cells), ' + ' (5-50\% positive cells) and ' ++ ' $(>50 \%$ positive cells). Only a nuclear expression pattern was considered as positive staining. For survival analysis, the DEK expression level was denoted as either positive expression (' + ' and ' ++ ') or negative expression ('-').

Statistical analyses. Statistical analyses were performed using SPSS 17.0. Correlations between DEK expression and clinicopathological characteristics were evaluated using $\chi^{2}$ and Fisher's exact tests. The disease-free and overall survival rates after tumor removal were calculated using the Kaplan-Meier method, and differences in survival curves were analyzed using log-rank tests. Multivariate survival analysis was performed on all significant characteristics measured by univariate survival analysis with the Cox proportional hazard regression model. A P-value of $<0.05$ was considered to indicate a statistically significant result.

\section{Results}

DEK expression in A549 cells and fresh tissues of NSCLC. DEK protein mainly localized to the nuclei of A549 cells by IF staining (Fig. 1). Moreover, the protein and mRNA expression levels of DEK were determined for 6 NSCLC samples with matched adjacent non-tumor fresh tissues. Western blot data showed that DEK protein was highly expressed in the NSCLC tissues compared with this level in the matched adjacent nontumor tissues (Fig. 2). qRT-PCR data confirmed an increased level of DEK mRNA expression in the NSCLC samples compared with that in the adjacent non-tumor tissues (Fig. 3).

DEK protein expression in paraffin-embedded NSCLC samples. DEK protein expression showed a strict nuclear staining pattern in NSCLC with immunohistochemistry, except that 3 cases of adenocarcinoma showed mainly a cytoplasmic staining pattern. DEK protein was negative in normal lung tissues, but usually upregulated in NSCLC. The positive 


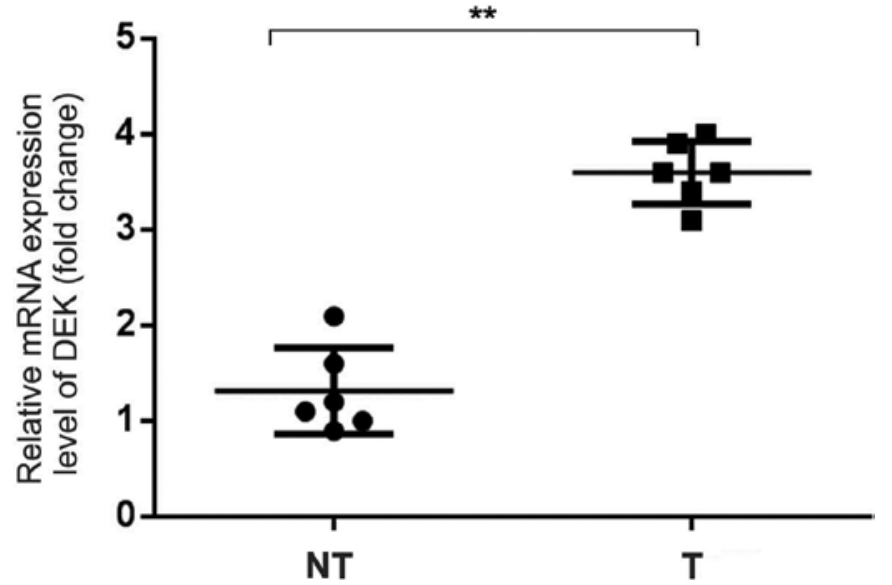

Figure 3. qRT-PCR analysis of DEK mRNA expression in fresh NSCLC (T) and non-cancerous tissue (NT) samples. Experiments were performed in triplicate for each case. DEK mRNA expression levels were significantly higher in NSCLC compared with adjacent non-tumor tissues ( $\left.{ }^{* *} \mathrm{P}<0.01\right)$.

rate of DEK protein was $66.33 \%$ (130/196) in NSCLC tissues, and was significantly higher than that in either adjacent nontumor tissues $(26.67 \%, 8 / 30)$ or normal lung tissues $(0 \%, 0 / 20)$ $(\mathrm{P}<0.001)$ (Fig. 4 and Table I).

Correlation between DEK expression and clinicopathological features of NSCLC. To evaluate the role of DEK protein in NSCLC progression, we analyzed correlations between DEK protein expression and major clinicopathological features of NSCLC. The results showed that DEK expression was significantly correlated to differentiation and clinical stages of the NSCLC cases $(\mathrm{P}=0.001$ and $\mathrm{P}=0.016$, respectively). However, DEK expression levels were not correlated to age, gender, tumor size, CEA level, smoking status, pathological subtype and metastasis of NSCLC ( $>>0.05)$ (Table II).

Correlation between the survival rates and DEK expression using the Kaplan-Meier method. To further confirm the role of DEK expression in NSCLC progression, we analyzed disease-free survival and overall survival rates of 196 NSCLC cases using the Kaplan-Meier method. We found that NSCLC patients with DEK expression had a lower disease-free survival rate (log-rank=11.704, $\mathrm{P}=0.001$ ) and a lower overall survival rate $(\log$-rank $=12.557, \mathrm{P}<0.001)$ than those patients without DEK expression (Fig. 5).

To substantiate the importance of DEK expression in NSCLC progression, we analyzed correlations between DEK expression and clinical stages of NSCLC. In earlystage NSCLC, patients with DEK expression had lower disease-free and overall survival rates compared with patients without DEK expression $(\mathrm{P}=0.004$ and $\mathrm{P}=0.003$, respectively) (Fig. 6A and $\mathrm{B}$ ). However, disease-free and overall survival rates were not correlated to DEK expression status $(\mathrm{P}=0.136$, and $\mathrm{P}=0.125$, respectively) in late-stage NSCLC (Fig. 6C and D).

DEK is an independent prognostic factor in NSCLC using the Cox proportional hazard regression model. Univariate analysis showed that patients with NSCLC tumors that expressed DEK had significantly lower overall survival $(\mathrm{P}=0.005)$ than patients with NSCLC tumors that did not express DEK. Additionally, patient age $(\mathrm{P}=0.043)$, pathological stage $(\mathrm{P}=0.000)$ and lymph node metastasis $(\mathrm{P}=0.001)$ were associated with the overall survival rate. Therefore, multivariate survival analysis was performed using the Cox proportional hazards model for all the significant variables found with univariate survival analysis. The results suggested that clinical stage (HR, 1.689; 95\% CI,

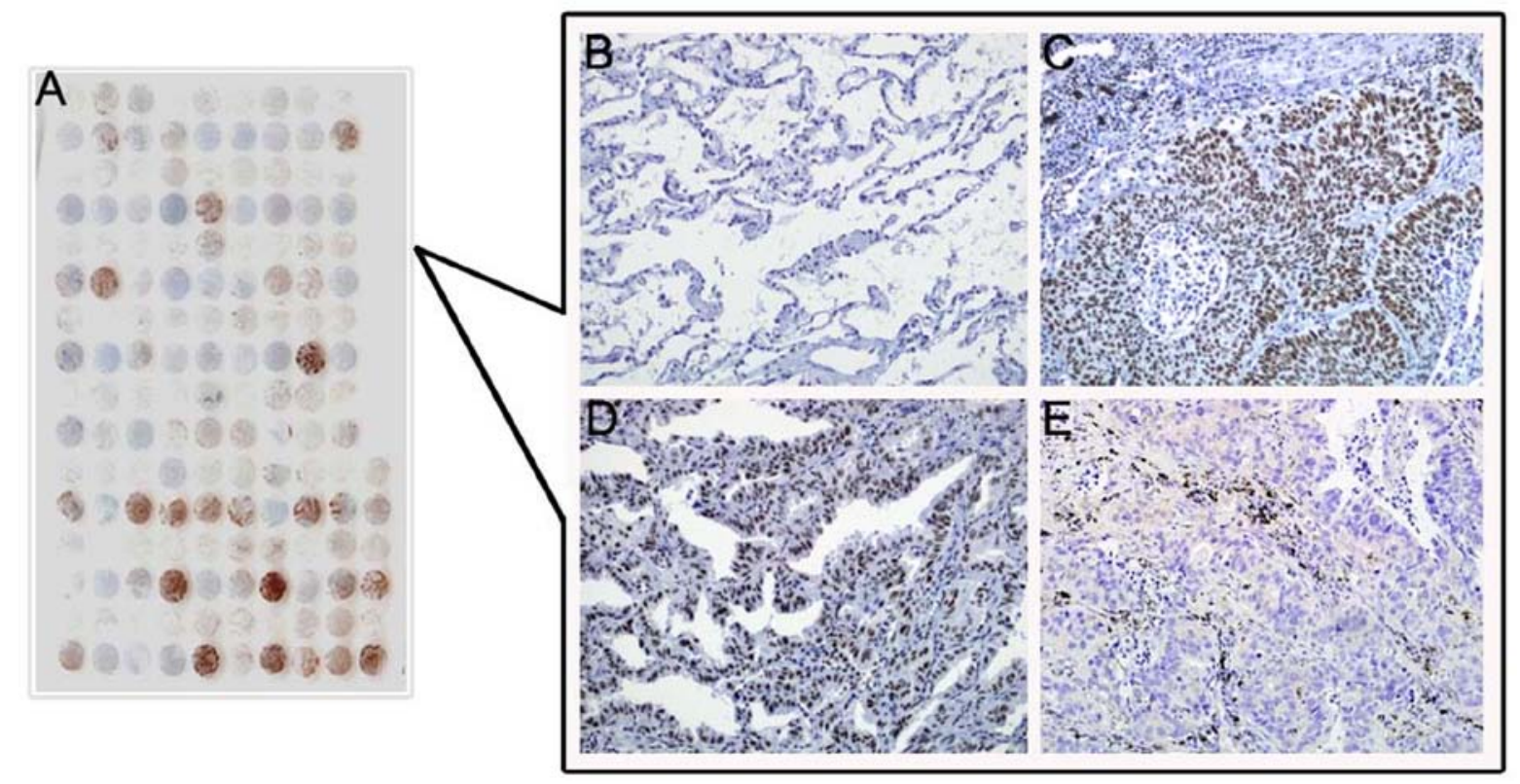

Figure 4. IHC staining of DEK in NSCLC tissue samples. (A) DEK protein staining in NSCLC tissue-array. (B) DEK is absolutely negative in normal lung tissue (original magnification, x200). (C) DEK is strongly positive in squamous cell carcinoma (SCC) with lymph node metastasis (original magnification, $\mathrm{x} 200$ ). (D) DEK is strongly positive in adenocarcinoms (original magnification, $\mathrm{x} 200$ ). (E) DEK is negative in SCC without lymph node metastasis (original magnification, $\mathrm{x} 200$ ). 
Table I. DEK protein expression in the NSCLC cases.

\begin{tabular}{lcccccc}
\hline & & \multicolumn{2}{c}{ DEK protein expression } & & \\
\cline { 3 - 5 } Tissues & No. of cases & - & + & ++ & Positive rate (\%) & P-value \\
\hline NSCLC & 196 & 66 & 51 & 79 & 66.33 & $0.000^{\mathrm{b}}$ \\
Adjacent non-tumor & 30 & 22 & 2 & 6 & 26.67 & $0.015^{\mathrm{a}}$ \\
Normal lung & 20 & 20 & 0 & 0 & 0 & \\
\hline
\end{tabular}

Statistical analyses were performed using Pearson's $\chi^{2}$ tests. ${ }^{\mathrm{a}} \mathrm{P}<0.05$ compared with normal lung tissues. ${ }^{\mathrm{b}} \mathrm{P}<0.01$ compared with adjacent non-tumor tissues. NSCLC, non-small cell lung carcinoma.

Table II. Correlation between DEK expression and clinicopathological features of the NSCLC cases.

\begin{tabular}{|c|c|c|c|c|}
\hline \multirow[b]{2}{*}{ Variables } & \multicolumn{2}{|c|}{ DEK protein expression (\%) } & \multirow[b]{2}{*}{$\chi^{2}$} & \multirow[b]{2}{*}{ P-value } \\
\hline & $+/++$ & - & & \\
\hline Age (years) & & & 0.081 & 0.776 \\
\hline$<65$ & $86(65.65)$ & $45(34.35)$ & & \\
\hline$\geq 65$ & $44(67.69)$ & $21(32.31)$ & & \\
\hline Gender & & & 0.677 & 0.412 \\
\hline Male & $75(68.81)$ & $34(31.19)$ & & \\
\hline Female & $55(63.22)$ & $32(36.78)$ & & \\
\hline Tumor size $(\mathrm{cm})$ & & & 0.535 & 0.466 \\
\hline$\leq 3$ & $52(63.41)$ & $30(39.59)$ & & \\
\hline$>3$ & $78(68.42)$ & $36(31.58)$ & & \\
\hline Differentiation & & & 12.210 & $0.001^{\mathrm{b}}$ \\
\hline Well & $20(45.45)$ & $24(54.55)$ & & \\
\hline Moderately & $65(69.15)$ & $29(30.85)$ & & \\
\hline Poorly & 45 (77.59) & $13(22.41)$ & & \\
\hline Pathological subtype & & & 1.982 & 0.160 \\
\hline SCC & $67(62.04)$ & $41(37.96)$ & & \\
\hline $\mathrm{AC}$ & $63(71.59)$ & $25(28.41)$ & & \\
\hline Clinical stage & & & 5.839 & $0.016^{\mathrm{a}}$ \\
\hline I-II & $59(58.42)$ & $42(41.58)$ & & \\
\hline III-IV & $71(74.74)$ & $24(25.26)$ & & \\
\hline LN metastasis & & & 1.420 & 0.235 \\
\hline Positive & $88(69.29)$ & $39(30.71)$ & & \\
\hline Negative & $42(60.87)$ & $27(39.13)$ & & \\
\hline CEA level & & & 2.589 & 1.108 \\
\hline Normal & $63(61.17)$ & $40(38.83)$ & & \\
\hline Increased & $67(72.04)$ & $26(27.96)$ & & \\
\hline Smoking status & & & 0.177 & 0.675 \\
\hline Yes & $101(65.58)$ & $53(34.42)$ & & \\
\hline No & $29(69.05)$ & $13(30.95)$ & & \\
\hline
\end{tabular}

Statistical analyses were performed using Pearson's $\chi^{2}$ square tests. ${ }^{\text {a }}<0.05,{ }^{b} \mathrm{P}<0.01$. NSCLC, non-small cell lung carcinoma; SCC, squamous cell carcinoma; AC, adenocarcinoma; LN, lymph node; CEA, carcinoembryonic antigen.

1.261-2.261; $\mathrm{P}<0.001)$, and lymph node metastasis (HR, 1.516; $95 \% \mathrm{CI}, 1.132-2.029 ; \mathrm{P}=0.005)$ were independent prognostic factors for overall survival rates in NSCLC. Importantly, DEK expression also emerged as a significant independent prognostic factor in the prognosis of NSCLC (HR, 1.388; 95\% CI, 1.024-1.882; $\mathrm{P}=0.035$ ) (Table III). 
A

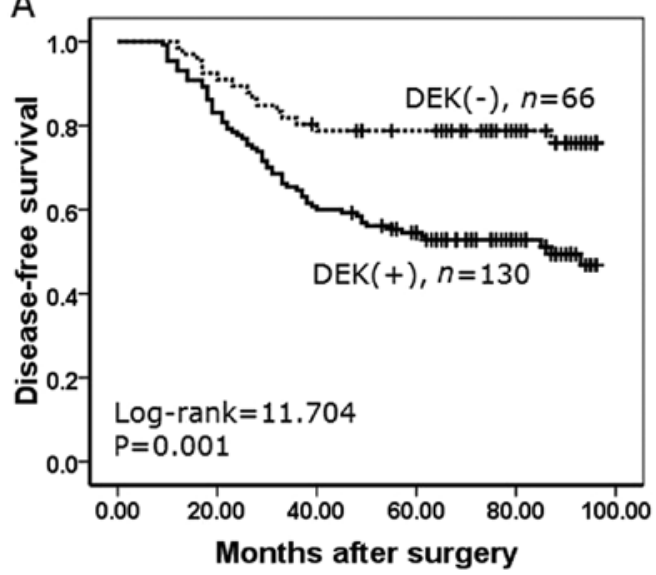

B

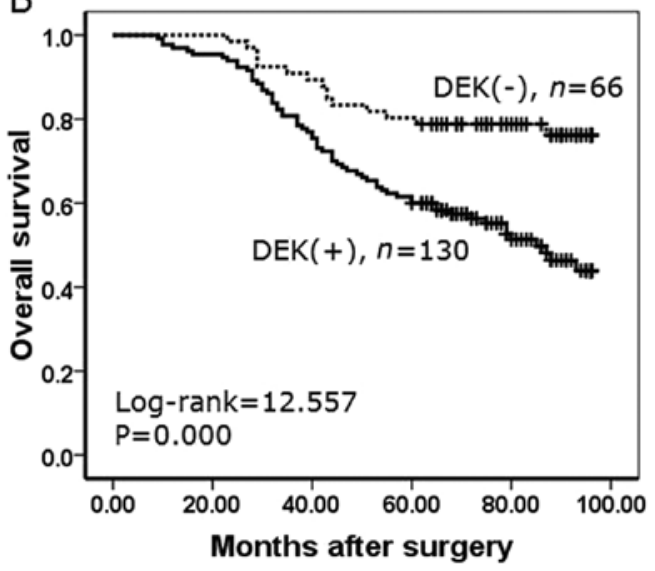

Figure 5. Kaplan-Meier analysis of disease-free and overall survival rates in 196 NSCLC patients in relation to DEK protein expression. (A) NSCLC patients with DEK-positive expression had a lower disease-free survival rate than those with DEK-negative expression $($ log-rank=11.704, $\mathrm{P}=0.001)$. (B) NSCLC patients with DEK-positive expression had a lower overall survival rate than those with DEK-negative expression $($ log-rank=12.557, $\mathrm{P}=0.000)(+$, positive; - , negative).

A

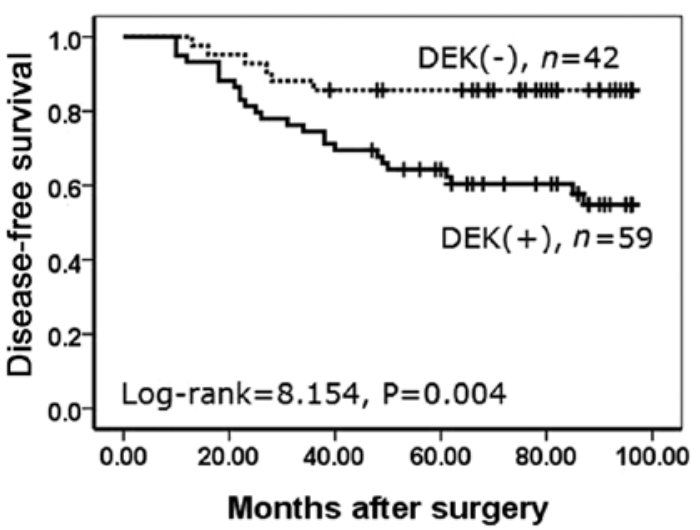

C

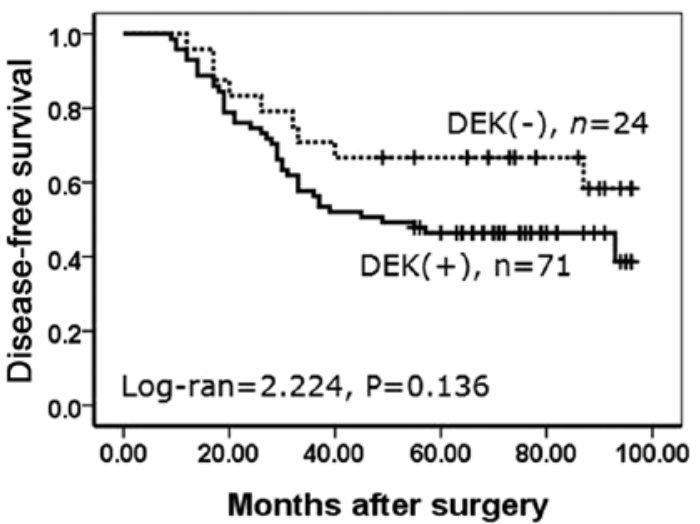

B

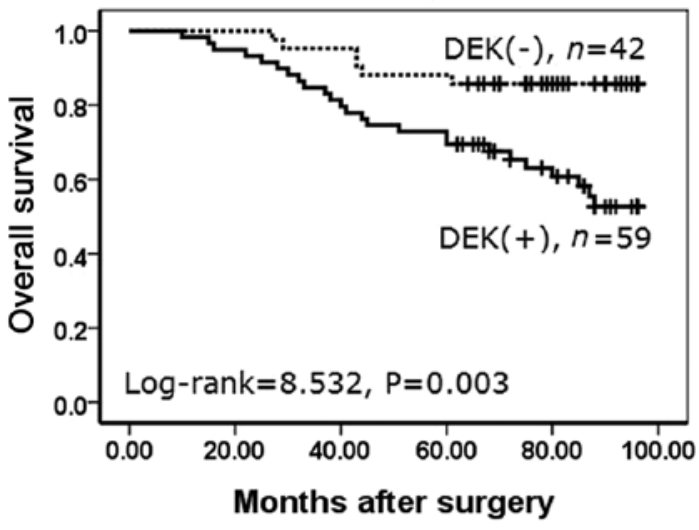

D

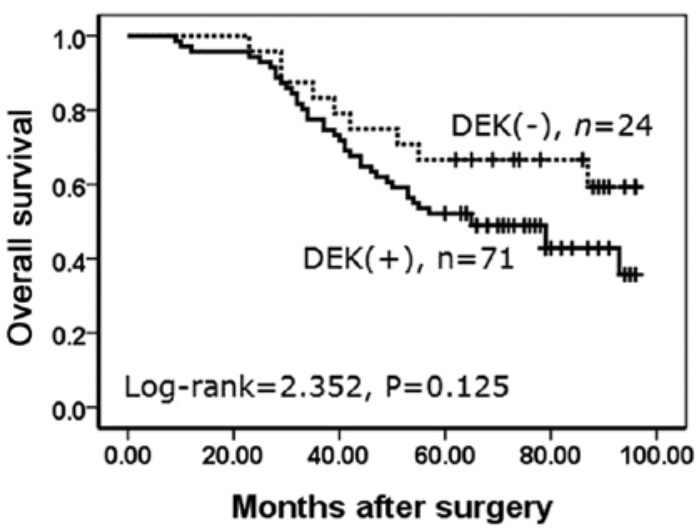

Figure 6. Kaplan-Meier analysis of disease-free survival and overall survival rates in 196 NSCLC patients with or without DEK expression in relation to clinical stage. (A) In the early-stage, disease-free survival rate of patients with DEK-positive expression was lower than the rate in patients with DEK-negative expression (log-rank=8.154, $\mathrm{P}=0.004)$. (B) In the early-stage, overall survival rate of patients with DEK-positive expression was lower than the rate in patients with DEK-negative expression (log-rank=8.532, $\mathrm{P}=0.003)$. (C) In the late-stage, disease-free survival rate of patients was not correlated with $\mathrm{DEK}$ expression (log-rank=2.224, $\mathrm{P}=0.136)$. (D) In the late-stage, overall survival rate of patients was not correlated with $\mathrm{DEK}$ expression $($ log-rank=2.352, $\mathrm{P}=0.125)(+$, positive; -, negative).

\section{Discussion}

DEK is located on chromosome $6 \mathrm{p} 22.3$, and was initially described as the target of a recurrent $t(6 ; 9)$ translocation in a subset of acute myeloid leukemia patients. Human DEK protein consists of 375 amino acids with four distinct trenches of acidic amino acids. As a highly conserved nuclear factor and the only member of its protein class, it is preferentially 
Table III. Univariate and multivariate survival analyses of the clinicopathological factors for the overall survival rate of 196 patients with NSCLC.

\begin{tabular}{|c|c|c|c|c|c|c|c|}
\hline \multirow[b]{2}{*}{ Characteristics } & \multirow[b]{2}{*}{$\beta$} & \multirow[b]{2}{*}{ SE } & \multirow[b]{2}{*}{ Wald } & \multirow[b]{2}{*}{ HR } & \multicolumn{2}{|c|}{$95 \% \mathrm{CI}$} & \multirow[b]{2}{*}{ P-value } \\
\hline & & & & & Lower & Upper & \\
\hline \multicolumn{8}{|l|}{ Univariate } \\
\hline Gender & 0.106 & 0.144 & 0.547 & 1.112 & 0.839 & 1.475 & 0.460 \\
\hline Age (years) & 0.309 & 0.152 & 4.102 & 1.361 & 1.010 & 1.835 & $0.043^{\mathrm{a}}$ \\
\hline Smoking status & 0.132 & 0.174 & 0.571 & 1.141 & 0.811 & 1.606 & 0.450 \\
\hline Tumor size & 0.279 & 0.146 & 3.663 & 1.322 & 0.993 & 1.761 & 0.056 \\
\hline Clinical stage & 0.589 & 0.147 & 16.090 & 1.801 & 1.351 & 2.401 & $0.000^{\mathrm{b}}$ \\
\hline Differentiation & 0.194 & 0.101 & 3.680 & 1.214 & 0.996 & 1.479 & 0.055 \\
\hline CEA & 0.030 & 0.143 & 0.044 & 1.030 & 0.778 & 1.364 & 0.834 \\
\hline Pathological subtype & 0.064 & 0.144 & 0.197 & 1.066 & 0.804 & 1.413 & 0.657 \\
\hline LN metastasis & 0.472 & 0.148 & 10.226 & 1.603 & 1.201 & 2.142 & $0.001^{\mathrm{b}}$ \\
\hline DEK & 0.423 & 0.152 & 7.739 & 1.527 & 1.133 & 2.057 & $0.005^{\mathrm{b}}$ \\
\hline \multicolumn{8}{|l|}{ Multivariate } \\
\hline Age (years) & 0.227 & 0.155 & 2.166 & 1.255 & 0.927 & 1.700 & 0.141 \\
\hline Clinical stage & 0.524 & 0.149 & 12.384 & 1.689 & 1.261 & 2.261 & $0.000^{\mathrm{a}}$ \\
\hline $\mathrm{LN}$ metastasis & 0.416 & 0.149 & 7.797 & 1.516 & 1.132 & 2.029 & $0.005^{\mathrm{a}}$ \\
\hline DEK & 0.328 & 0.155 & 4.458 & 1.388 & 1.024 & 1.882 & $0.035^{\mathrm{a}}$ \\
\hline
\end{tabular}

Statistical analyses were performed using Cox proportional hazard regression model. ${ }^{\mathrm{a}} \mathrm{P}<0.05,{ }^{\mathrm{b}} \mathrm{P}<0.01$. NSCLC, non-small cell lung carcinoma; CI, confidence interval; CEA, carcinoembryonic antigen; LN, lymph node.

expressed in actively proliferating and malignant cells, where it can reach up to 4 to 6 million copies/nucleus $(16,17)$.

The ability of DEK to bind nucleic acid has led to functional associations with several cellular processes, including chromatin remodeling, transcriptional regulation, replication, mRNA splicing and DNA repair (18-21). Carro et al found that when DEK is upregulated, as observed in numerous types of cancer, perturbations to the normal genome architecture and integrity are likely contributors to oncogenesis (22). Privette Vinnedge et al reported that DEK depletion can result in cell death and impaired DNA double-strand break repair (9). Therefore, cellular DEK expression is tightly controlled to maintain proper cell function and viability. Wise-Draper et al found that there was a significant delay in the formation of papillomas in DEK-knockout mice compared with wild-type and heterozygous littermate mice (5). Papillomas ultimately formed in the DEK-knockout mice suggesting a role for DEK in tumor initiation in this model.

The increasing list of tumor types, including acute myeloid leukemia (23), glioblastoma (24), cervical cancer $(25,26)$, melanoma (22) and ovarian cancer (11) among others (27-29), showing high DEK protein expression raises the exciting possibility of using DEK as a tumor marker (29). Datta et al reported that DEK protein was present in voided urine of patients with both low- and high-grade bladder cancers, suggesting that DEK could be used as a biomarker for detection of this cancer using patient urine samples (30). Privette Vinnedge et al suggested that DEK promotes the pathogenesis of $\mathrm{ER}^{+}$breast cancer and that the targeted inhibition of DEK may enhance the efficacy of conventional hormone therapies (6). Our previous study showed that DEK protein expression was closely related to the disease-free and overall survival rates of patients with colorectal cancer, and its overexpression was an independent risk factor for mortality in colorectal cancer (13).

Few studies to date have reported an association between DEK expression and clinicopathological parameters, as well as the DEK prognostic role in lung cancer. Wang et al analyzed DEK immunohistochemistry in 112 NSCLC cases and reported that DEK-positive tumors were correlated with poor differentiation, advanced p-TNM stage and nodal metastasis, and DEK expression in lung adenocarcinoma was significantly higher compared with DEK expression in squamous cell carcinoma (31). In the present study, qRT-PCR and western blot data demonstrated that the levels of DEK mRNA and protein were significantly higher in NSCLC samples compared with adjacent non-tumor tissues. Furthermore, we performed immunohistochemical staining and analysis in 196 cases of NSCLC, and found that the positive rate of DEK protein expression was $66.33 \%$ in NSCLC, and was significantly higher than that noted in either adjacent non-tumor or normal lung tissues, indicating that DEK potentially plays an important role in the progression of NSCLC. These findings are consistent with the current theory that suggests that DEK upregulation is high in proliferating cells and low in resting and terminally differentiated cells $(4,32)$.

We examined DEK expression and the clinicopathological features of NSCLC and found that DEK expression was significantly correlated with poor differentiation $(\mathrm{P}<0.01)$ and advanced clinical stage $(\mathrm{P}<0.05)$, but was not related to age, gender, tumor size, nodal status, pathological subtype, 
CEA level and the smoking status of patients with NSCLS $(\mathrm{P}>0.05)$. However, Wang et al reported that DEK expression was significantly related to nodal metastasis and pathological subtype (31). The causes for the differences in results may be due to case selection, the source of the antibody used or staining method. Further study is needed to explore the mechanisms of DEK upregulation in NSCLC progression.

With regard to survival, we found that NSCLC patients with DEK expression had a lower disease-free survival rate $(\mathrm{P}=0.001)$ and overall survival rate $(\mathrm{P}<0.001)$ than patients without DEK expression. In early-stage NSCLC, patients with DEK expression had lower disease-free and overall survival rates compared with those without DEK expression ( $\mathrm{P}=0.004$ and $\mathrm{P}=0.003$, respectively). However, DEK expression status was not related to the survival of NSCLC patients with an advanced clinical stage. Multivariate survival analysis demonstrated that DEK expression emerged as a significantly independent hazard factor for overall survival in NSCLC, along with clinical stage and metastasis.

In conclusion, DEK plays an important role in NSCLC progression, and it may be an independent biomarker for evaluating prognosis in patients with NSCLC.

\section{Acknowledgements}

The present study was supported by grants from the National Natural Science Fund of China (no. 81272927), the Projects for Research and Innovation of the Jilin Youth Leader and Team (no. 20130521017JH), and the Projects of Science Research of the Education Department in Liaoning Province (no. L2015189).

\section{References}

1. Jemal A, Siegel R, Ward E, Murray T, Xu J and Thun MJ: Cancer statistics, 2007. CA Cancer J Clin 57: 43-66, 2007.

2. Brambilla E, Travis WD, Colby TV, Corrin B and Shimosato Y: The new World Health Organization classification of lung tumours. Eur Respir J 18: 1059-1068, 2001.

3. Lee HW, Kim EH and Oh MH: Clinicopathologic implication of ezrin expression in non-small cell lung cancer. Korean $\mathrm{J}$ Pathol 46: 470-477, 2012.

4. von Lindern M, Fornerod M, van Baal S, Jaegle M, de Wit T, Buijs A and Grosveld G: The translocation (6;9), associated with a specific subtype of acute myeloid leukemia, results in the fusion of two genes, dek and can, and the expression of a chimeric, leukemia-specific dek-can mRNA. Mol Cell Biol 12: 1687-1697, 1992.

5. Wise-Draper TM, Mintz-Cole RA, Morris TA, Simpson DS, Wikenheiser-Brokamp KA, Currier MA, Cripe TP, Grosveld GC and Wells SI: Overexpression of the cellular DEK protein promotes epithelial transformation in vitro and in vivo. Cancer Res 69: 1792-1799, 2009.

6. Privette Vinnedge LM, Ho SM, Wikenheiser-Brokamp KA and Wells SI: The DEK oncogene is a target of steroid hormone receptor signaling in breast cancer. PLoS One 7: e46985, 2012.

7. Cleary J, Sitwala KV, Khodadoust MS, Kwok RP, Mor-Vaknin N, Cebrat M, Cole PA and Markovitz DM: p300/CBP-associated factor drives DEK into interchromatin granule clusters. J Biol Chem 280: 31760-31767, 2005.

8. Khodadoust MS, Verhaegen M, Kappes F, Riveiro-Falkenbach E, Cigudosa JC, Kim DS, Chinnaiyan AM, Markovitz DM and Soengas MS: Melanoma proliferation and chemoresistance controlled by the DEK oncogene. Cancer Res 69: 6405-6413, 2009.

9. Privette Vinnedge LM, McClaine R, Wagh PK, WikenheiserBrokamp KA, Waltz SE and Wells SI: The human DEK oncogene stimulates $\beta$-catenin signaling, invasion and mammosphere formation in breast cancer. Oncogene 30: 2741-2752, 2011.
10. Hasiów-Jaroszewska B, Borodynko $\mathrm{N}$ and Pospieszny $\mathrm{H}$ : Infectious RNA transcripts derived from cloned cDNA of a pepino mosaic virus isolate. Arch Virol 154: 853-856, 2009.

11. Han S, Xuan Y, Liu S, Zhang M, Jin D, Jin R and Lin Z: Clinicopathological significance of DEK overexpression in serous ovarian tumors. Pathol Int 59: 443-447, 2009.

12. Wu Q, Li Z, Lin H, Han L, Liu S and Lin Z: DEK overexpression in uterine cervical cancers. Pathol Int 58: 378-382, 2008.

13. Lin L, Piao J, Gao W, Piao Y, Jin G, Ma Y, Li J and Lin Z: DEK over expression as an independent biomarker for poor prognosis in colorectal cancer. BMC Cancer 13: 366, 2013.

14. Liu S, Wang X, Sun F, Kong J, Li Z and Lin Z: DEK overexpression is correlated with the clinical features of breast cancer. Pathol Int 62: 176-181, 2012.

15. Wrona A and Jassem J: The new TNM classification in lung cancer. Pneumonol Alergol Pol 78: 407-417, 2010 (In Polish).

16. Kappes F, Burger K, Baack M, Fackelmayer FO and Gruss C: Subcellular localization of the human proto-oncogene protein DEK. J Biol Chem 276: 26317-26323, 2001.

17. Kappes F, Scholten I, Richter N, Gruss C and Waldmann T: Functional domains of the ubiquitous chromatin protein DEK. Mol Cell Biol 24: 6000-6010, 2004.

18. Alexiadis V, Waldmann T, Andersen J, Mann M, Knippers R and Gruss C: The protein encoded by the proto-oncogene DEK changes the topology of chromatin and reduces the efficiency of DNA replication in a chromatin-specific manner. Genes Dev 14: 1308-1312, 2000.

19. Campillos M, García MA, Valdivieso F and Vázquez J: Transcriptional activation by AP-2alpha is modulated by the oncogene DEK. Nucleic Acids Res 31: 1571-1575, 2003.

20. Soares LM, Zanier K, Mackereth C, Sattler M and Valcárcel J: Intron removal requires proofreading of $\mathrm{U} 2 \mathrm{AF} / 3^{\prime}$ splice site recognition by DEK. Science 312: 1961-1965, 2006.

21. Kavanaugh GM, Wise-Draper TM, Morreale RJ, Morrison MA, Gole B, Schwemberger S, Tichy ED, Lu L, Babcock GF, Wells JM, et al: The human $D E K$ oncogene regulates DNA damage response signaling and repair. Nucleic Acids Res 39: 7465-7476, 2011.

22. Carro MS, Spiga FM, Quarto M, Di Ninni V, Volorio S, Alcalay $\mathrm{M}$ and Müller H: DEK Expression is controlled by E2F and deregulated in diverse tumor types. Cell Cycle 5: 1202-1207, 2006.

23. Casas S, Nagy B, Elonen E, Aventín A, Larramendy ML, Sierra J, Ruutu T and Knuutila S: Aberrant expression of HOXA9, DEK, CBL and CSF1R in acute myeloid leukemia. Leuk Lymphoma 44: 1935-1941, 2003.

24. Kroes RA, Jastrow A, McLone MG, Yamamoto H, Colley P, Kersey DS, Yong VW, Mkrdichian E, Cerullo L, Leestma J, et al: The identification of novel therapeutic targets for the treatment of malignant brain tumors. Cancer Lett 156: 191-198, 2000.

25. Kappes F, Khodadoust MS, Yu L, Kim DS, Fullen DR, Markovitz DM and Ma L: DEK expression in melanocytic lesions. Hum Pathol 42: 932-938, 2011.

26. Liu K, Feng T, Liu J, Zhong M and Zhang S: Silencing of the DEK gene induces apoptosis and senescence in CaSki cervical carcinoma cells via the up-regulation of NF- $\kappa \mathrm{B}$ p65. Biosci Rep 32: 323-332, 2012.

27. Paderova J, Orlic-Milacic M, Yoshimoto M, da Cunha Santos G, Gallie B and Squire JA: Novel 6p rearrangements and recurrent translocation breakpoints in retinoblastoma cell lines identified by spectral karyotyping and mBAND analyses. Cancer Genet Cytogenet 179: 102-111, 2007.

28. Privette Vinnedge LM, Kappes F, Nassar N and Wells SI: Stacking the DEK: From chromatin topology to cancer stem cells. Cell Cycle 12: 51-66, 2013.

29. Lin L, Piao J, Ma Y, Jin T, Quan C, Kong J, Li Y and Lin Z: Mechanisms underlying cancer growth and apoptosis by DEK overexpression in colorectal cancer. PLoS One 9: e111260, 2014.

30. Datta A, Adelson ME, Mogilevkin Y, Mordechai E, Sidi AA and Trama JP: Oncoprotein DEK as a tissue and urinary biomarker for bladder cancer. BMC Cancer 11: 234, 2011.

31. Wang J, Sun L, Yang M, Luo W, Gao Y, Liu Z, Qiu X and Wang E: DEK depletion negatively regulates Rho/ROCK/MLC pathway in non-small cell lung cancer.J Histochem Cytochem 61: 510-521, 2013.

32. Kappes F, Damoc C, Knippers R, Przybylski M, Pinna LA and Gruss C: Phosphorylation by protein kinase CK 2 changes the DNA binding properties of the human chromatin protein DEK. Mol Cell Biol 24: 6011-6020, 2004. 